\title{
ADM Worldvolume Geometry
}

\author{
R. Capovilla ${ }^{\mathrm{a} *}$, J. Guven $^{\mathrm{b}}$ and E. Rojas ${ }^{\mathrm{a}}$ \\ aFísica, CINVESTAV-IPN, Apdo. Postal 14-740, 07000 México D.F., México \\ bInstituto de Ciencias Nucleares, Universidad Nacional Autónoma de México, Apdo. Postal 70-543, \\ 04510 México D.F., México
}

\begin{abstract}
We describe the dynamics of a relativistic extended object in terms of the geometry of a configuration of constant time. This involves an adaptation of the ADM formulation of canonical general relativity. We apply the formalism to the hamiltonian formulation of a Dirac-Nambu-Goto [DNG] relativistic extended object in an arbitrary background spacetime.
\end{abstract}

The mechanics of relativistic extended objects, or branes for short, has been the subject of various investigations in recent years. In particular, the usefulness of a geometrical approach which mantains general covariance with respect to worldvolume reparametrizations and rotations of worldvolume normal vector fields has become apparent (see e.g. [1,2]). However, this geometrical approach has been developed largely within the lagrangian formulation of theories of relativistic extended objects. The geometry of interest is then the worldvolume geometry. In this note, we report on an alternative approach where we focus on the geometry of the relativistic extended object itself, and its relation with the worldvolume geometry [3]. One immediate application of this formalism is a geometrical Hamiltonian formulation of this type of theories, which mantains worldvolume covariance without resorting to gauge fixing from the outset. (For alternative treatments see e.g. [4 (6).

We consider a relativistic extended object $\Sigma$, of dimension $d$, embedded in an arbitrary fixed $(N+$ 1)-dimensional background spacetime $\left\{M, g_{\mu \nu}\right\}$. $\Sigma$ is described locally by the spacelike embedding $x^{\mu}=X^{\mu}\left(u^{A}\right)$, where $x^{\mu}$ are local coordinates for the background spacetime, $u^{A}$ local coordinates for $\Sigma$, and $X^{\mu}$ the embedding functions $(\mu, \nu, \cdots=0,1, \cdots, N$, and $A, B, \cdots=1, \cdots, d)$. The tangent vectors to $\Sigma$ are defined by $\epsilon_{A}:=$

\footnotetext{
* Supported in part by the organizers and by the Physics Department of CINVESTAV-IPN.
}

$\left(\partial_{A} X^{\mu}\right) \partial_{\mu}$, so that the positive-definite metric induced on $\Sigma$ is

$h_{A B}:=g\left(\epsilon_{A}, \epsilon_{B}\right)$.

We construct out of the metric $h_{A B}$ the intrinsic geometry of $\Sigma$. Note that it will be trivial in the special case of a relativistic string, since then $\Sigma$ is one-dimensional.

For the extrinsic geometry of $\Sigma$, we introduce the normals $\left\{m^{I}\right\}$, defined by $g\left(\epsilon_{A}, m^{I}\right)=0$, and normalized with $g\left(m^{I}, m^{J}\right)=\eta^{I J}$, where $\eta_{I J}$ is the Minkowski metric, with only one minus sign $(I, J, \cdots=0,1, \cdots, N+1-d)$. The extrinsic curvature along the $I$-th normal is

$L_{A B}{ }^{I}:=-g\left(m^{I}, D_{A} \epsilon_{B}\right)=L_{B A}{ }^{I}$,

where $D_{A}=\epsilon_{A}^{\mu} D_{\mu}$ is the spacetime covariant derivative compatible with $g_{\mu \nu}$ along the tangent directions. In addition, the extrinsic geometry of $\Sigma$ is determined by the extrinsic twist, $\sigma_{A}{ }^{I J}$, defined by

$\sigma_{A}^{I J}:=g\left(m^{J}, D_{A} m^{I}\right)=-\sigma_{A}^{J I}$.

This is a connection associated with the $O(N+1-$ d) freedom in the definition of the normal fields. In the mathematical literature it is known as the normal form. When the appropriate GaussCodazzi-Mainardi integrability conditions hold, $\left\{h_{A B}, L_{A B}{ }^{I}, \sigma_{A}{ }^{I J}\right\}$ define the geometry of $\Sigma$.

We consider now the time evolution of $\Sigma$ in spacetime. We denote its trajectory, or worldvolume, by $w$. It is an oriented timelike surface 
in spacetime. Now the shape functions become time-dependent, $X^{\mu}=X^{\mu}\left(t, u^{A}\right)$, where $t$ is a coordinate that labels the leafs of the foliation of $w$ by $\Sigma$ s.

From the point of view of an observer sitting on $\Sigma$, this involves breaking the normal rotation symmetry of $\Sigma, O(N+1-d)$, down to $O(N-$ $d$ ), by choosing the unit (future-pointing) timelike normal to $\Sigma$ into $w, m^{0}=: \eta$. We denote by $m^{i}=$ : $n^{i}$ the remaining components of $\left\{m^{I}\right\}(i, j, \cdots=$ $1,2, \cdots, N-d)$. These are also normals to the worldvolume $w$. This symmetry reduction is the key ingredient in this geometrical construction.

The time evolution of the embedding functions for $\Sigma$ into the worldvolume can be written as,

$\dot{X}^{\mu}:=N \eta^{\mu}+N^{A} \epsilon^{\mu}{ }_{A}$,

where, following standard usage in general relativity, $N$ is called the lapse function, and $N^{A}$ the shift vector. We emphasize that the content of this equation is simply that the time evolution of $\Sigma$ is into the worldvolume $w$. Note that we can always chose a time evolution normal to $\Sigma$, i.e. take the shift vector to vanish, $N^{A}=0$ (see e.g. (5).

At this point, we introduce the geometry of the worldvolume $w$. The worldvolume can be represented in parametric form by the embedding functions $x^{\mu}=\chi^{\mu}\left(\xi^{a}\right)$, where $\xi^{a}=\left\{t, u^{A}\right\}$ are local coordinates for $w$, and $\chi^{\mu}$ the embedding functions $(a, b, \cdots=0,1, \cdots, d)$.

The tangent vectors to $w, e_{a}:=e^{\mu}{ }_{a} \partial_{\mu}=$ $\left(\partial_{a} \chi^{\mu}\right) \partial_{\mu}$, decompose in a part tangential to $\Sigma$ and a part along the time evolution of $\Sigma$,

$e^{\mu}{ }_{a}=\left(\begin{array}{c}\dot{X}^{\mu} \\ \epsilon_{A}^{\mu}\end{array}\right)$.

It follows that the lorentzian worldvolume induced metric, $\gamma_{a b}:=g\left(e_{a}, e_{b}\right)$, decomposes according to the familiar ADM expression as,

$\gamma_{a b}=\left(\begin{array}{cc}-N^{2}+N^{A} N^{B} h_{A B} & h_{A B} N^{B} \\ h_{A B} N^{B} & h_{A B}\end{array}\right)$.

Note that the worldvolume element is given by $\sqrt{-\gamma}=N \sqrt{h}$. The various geometrical quantities that characterize the intrinsic geometry of $\Sigma$, such as its Riemann curvature etc., can be de- composed by importing the appropriate expressions from the ADM treatment of spacetime in canonical general relativity.

It is worth emphasizing the difference between, say, the lapse function in this context versus the lapse function in canonical general relativity. Whereas here it is a component of the velocity, in the latter case it is a component of the metric.

The extrinsic curvature of the worldvolume $w$ along the $i$-th normal vector field $\left\{n^{i}\right\}$ is defined by

$K_{a b}{ }^{i}:=-g\left(n^{i}, D_{a} e_{b}\right)=K_{b a}^{i}$,

where $D_{a}=e^{\mu}{ }_{a} D_{\mu}$ is the gradient along the vectors tangential to $w$. It can be decomposed as

$K_{a b}{ }^{i}=\left(\begin{array}{cc}-n_{\mu}{ }^{i} \ddot{X}^{\mu} & H_{A}{ }^{i} \\ H_{A}{ }^{i} & L_{A B}{ }^{i}\end{array}\right)$,

where we have introduced the quantity

$H_{A}{ }^{i}:=N \sigma_{A}^{0 i}+N^{B} L_{A B}^{i}$.

We note that the time-time component of the extrinsic curvature is (minus) the projection into the normals of the acceleration of $\Sigma_{t}$. For the degenerate case of a relativistic particle, this is all there is. The off-diagonal components involve the extrinsic twist of $\Sigma$. This is a consequence of having broken the full normal rotation symmetry. The spatial components involve the extrinsic curvature of $\Sigma$ along the normals $\left\{n^{i}\right\}$

The ADM decomposition of the mean extrinsic curvature of the worldvolume, $K^{i}:=\gamma^{a b} K_{a b}{ }^{i}$, is readily obtained as

$$
\begin{aligned}
K^{i} & =N^{-2}\left[n_{\mu}{ }^{i} \ddot{X}^{\mu}+2 N^{A} H_{A}{ }^{i}\right. \\
& \left.-N^{A} N^{B} L_{A B}^{i}+N^{2} L^{i}\right],
\end{aligned}
$$

where $L^{i}=h^{A B} L_{A B}{ }^{i}$ is the mean extrinsic curvature of $\Sigma$ along the normals to the worldvolume $\left\{n^{i}\right\}$. This is the generalization to an extended object of the acceleration for a relativistic particle.

We can put the formalism we have developed to use in the analysis of the dynamics of a relativistic extended object. For example, for a simple DNG object, which extremizes the worldvolume, the equations of motion are given by the vanishing of the mean extrinsic curvature,

$K^{i}=0$. 
If we specialize to normal time evolution, use of the expression (10) allows us to rewrite this equation in the form,

$n_{\mu}{ }^{i} \ddot{X}^{\mu}+N^{2} L^{i}=0$.

This identifies a part of the extrinsic curvature of $\Sigma$ as the driving force in its dynamics. We emphasize that these expressions hold in an arbitrary background spacetime.

The natural application of this geometrical approach is the Hamiltonian formulation of a theory of relativistic extended objects. For simplicity, we consider here only the case of a DNG object. Although the benefits of a geometrical approach are already apparent in this case, the full power of the formalism is displayed when considering higher-order curvature dependent actions [7], or additional worldvolume fields as for example in the case of superconducting membranes [8].

We write the DNG action as $S=\int d t L[X, \dot{X}]$, where, with $\mu$ the brane tension, the lagrangian is

$$
\begin{aligned}
L[X, \dot{X}] & =-\mu \int_{\Sigma} d^{d} u \sqrt{-\gamma} \\
& =-\mu \int_{\Sigma} d^{d} u N \sqrt{h} .
\end{aligned}
$$

Therefore, the lapse function plays the role of the lagrangian density for a DNG brane. The canonical momenta are given by $P_{\mu}:=\delta L / \delta \dot{X}^{\mu}$. Using the definition (雨) for the lapse function, we find

$P_{\mu}=\mu \sqrt{h} \eta_{\mu}$.

The factor of $\sqrt{h}$ comes as no surprise - momenta are densities. The "shape" functions of $\Sigma$ are the configuration variables, and their conjugate momenta are proportional to the unit normal of $\Sigma$ into the worldvolume. The phase space for our extended object is then naturally associated with the geometry of $\Sigma$.

As expected from worldvolume reparametrization invariance, the hamiltonian vanishes, $H[X, P]=\int d^{d} u\left[P_{\mu} \dot{X}^{\mu}\right]-L[X, \dot{X}]=0$. However, according to the standard Dirac treatment of constrained systems, the hamiltonian is a linear combination of the phase space constraints that generate reparametrizations $\left\{\mathcal{C}_{0}, \mathcal{C}_{A}\right\}$, The explicit form of the constraints is easily obtained from the definition of the momenta as

$$
\begin{aligned}
\mathcal{C}_{A}(X, P) & =P_{\mu} \epsilon^{\mu}{ }_{A}=0, \\
\mathcal{C}_{0}(X, P) & =P^{2}+\mu^{2} h=0,
\end{aligned}
$$

and the hamiltonian is

$H[X, P]=\int_{\Sigma_{t}}\left[\lambda \mathcal{C}_{0}+\lambda^{A} \mathcal{C}_{A}\right]$

where $\lambda, \lambda^{A}$ are lagrange multipliers. Note that the lagrange multiplier $\lambda$ must be a scalar density of weight -1 for the hamiltonian to be welldefined.

The first constraint, or vector constraint, is universal for all reparametrizations invariant actions. Clearly it has no analogue in the case of a relativistic particle, when $\Sigma$ degenerates to a point. It is easy to see that it is the generator of spatial diffeomorphisms.

The second, or scalar constraint, is the generalization to extended objects of the familiar hamiltonian constraint for a parametrized massive relativistic particle. It generates the evolution of $\Sigma$. For a relativistic string, it is possible to exploit the triviality of its one-dimensional $\Sigma$, by choosing proper length as the parameter along the string, so that $h=1$, the potential is constant, and the scalar constraint takes the form $P^{2}+\mu^{2}=0$. This simple observation explains the integrability of the string in a variety of background spacetimes (see e.g. 9). (It also strongly suggests that the string may be integrable in any background spacetime.) Already for a membrane $(d=2)$, however, such a priviledged parametrization for $\Sigma$ is not available. The determinant of $h_{A B}$ contains four powers of (spatial derivatives of) $X^{\mu}$, and the theory is highly non-linear, even in a flat background. Hopes of integrability are evidently slim. Introducing the bracket [10,11]

$\left\{X^{\mu}, X^{\nu}\right\}=\epsilon^{A B} \epsilon^{\mu}{ }_{A} \epsilon_{B}^{\nu}$,

we can rewrite the scalar constraint for a relativistic membrane in the form

$P^{2}+\mu^{2}\left\{X^{\mu}, X^{\nu}\right\}^{2}=0$.

In this form we can make contact with current approaches to the canonical formulations of membrane dynamics (see [11,6] ). 
The constraints are first class. The Poisson algebra of the constraint functions reproduce the familiar algebra of hypersurface deformation for $\Sigma$, now seen as a hypersurface in the worldvolume $w$ 12. In general it is not a Lie algebra since the structure "constants" depend on the configuration variables via the (densitized) metric $h h^{A B}$ in the scalar-scalar Poisson bracket. This is familiar from the constraint algebra of general relativity. However, for a relativistic string, the triviality of the intrinsic geometry of $\Sigma$ allows to set $h h^{A B}=1$, and we have a true Lie algebra, which can be shown to be isomorphic to the algebra of the conformal group in two dimensions [13].

Let us consider the Hamilton equations. We have that

$\dot{X}^{\mu}=2 \lambda P^{\mu}+\lambda^{A} \epsilon^{\mu}{ }_{A}$.

It reproduces the definition of the momentum (14), and it identifies $\lambda^{A}=N^{A}, \lambda=N / 2 \mu \sqrt{h}$, assuming that $P_{\mu}$ is future-pointing. Using this information, the second Hamilton equation is

$$
\begin{aligned}
\dot{P}_{\mu} & =\frac{1}{2} h^{-1} \dot{h} P_{\mu}-N \mu \sqrt{h} L^{i} n_{\mu i} \\
& +\mu \sqrt{h}\left(\mathcal{D}_{A} N\right) \epsilon_{\mu}{ }^{A}+\mathcal{D}_{A}\left(N^{A} P_{\mu}\right),
\end{aligned}
$$

where, for convenience, we have left $\dot{h}$ in its implicit form. Note that $\dot{h}=2 N h k$, where $k=h^{A B} L_{A B}{ }^{0}$ is the mean extrinsic curvature of $\Sigma$ embedded as a hypersurface in $w$. For the analysis of this equation it is convenient to take $N^{A}=0$, and the shift constant $\mathcal{D}_{A} N=0$. The projection along the momentum is a mere identity,

$\dot{P}_{\mu} P^{\mu}=\frac{1}{2} h^{-1} \dot{h} P^{2}$,

as can be seen using the scalar constraint, and its time derivative. The normal projection

$\dot{P}_{\mu} n^{\mu}{ }_{i}=-N \mu \sqrt{h} L_{i}$,

then reproduces the equations of motion in the form (11).

This geometrical approach to the canonical formulation of relativistic extended objects provides valuable insight into their dynamics, and when it is relevant, into their canonical quantization. Furthermore, placing their description on the same footing as canonical general relativity has the potential to reap mutual benefits, both technically and conceptually.

\section{REFERENCES}

1. B. Carter, in Formation and Interaction of Topological Defects, eds. A.C. Davis and R. Brandenberger (Plenum Press, New York, 1995).

2. R. Capovilla and J. Guven, Phys. Rev. D 51 (1995) 6736.

3. R. Capovilla, J. Guven and E. Rojas, preprint.

4. P.A. Collinson, J.F.L. Hopkinson and R.W. Tucker, Nucl. Phys. B 100 (1975) 157.

5. J. Hoppe, hep-th/9407103.

6. L. Smolin, Phys. Rev. D 57 (1998) 6216.

7. R. Capovilla, J. Guven and E. Rojas, in preparation.

8. R. Cordero and E. Rojas, (to appear in Phys. Lett. B)

9. I. Bakas and K. Sfetsos, Phys. Rev. D 54 (1996) 3995.

10. J. Hoppe, PhD thesis, MIT (1982).

11. H. Nicolai and R. Helling, hep-th/9809103.

12. C. Teitelboim, Ann. Phys. 79 (1973) 542.

13. K. Kuchar and C. G. Torre, J. Math. Phys. 30 (1989) 1769. 